\section{International Scientific Journal Theoretical \& Applied Science}

\author{
p-ISSN: 2308-4944 (print) e-ISSN: 2409-0085 (online) \\ Year: 2017 Issue: $06 \quad$ Volume: 50 \\ Published: 17.06.2017 http://T-Science.org
}

SECTION 7. Mechanics and machine construction
Gayrat Ataxanovich Bahadirov

Doc. of Tech. Sc., Chief researcher, Institute of Mechanics and Seismic Stability of Structures named after M.T.Urazbaev of the Academy of Sciences of the Republic of Uzbekistan, Tashkent instmech@rambler.ru

Nusratilla Radjabovich Barakaev Doc. of Tech. Sc., Pro-rector Gulistan state university, Gulistan

Abdusalam Abdukarimov

Cand. of Tech. Sc., Head of the Chair, Institute of Mechanics and Seismic Stability of Structures named after M.T.Urazbaev of the Academy of Sciences of the Republic of Uzbekistan, Tashkent

Abdurasul Abdurahimovich Umarov Junior researcher, Institute of Mechanics and Seismic Stability of Structures named after M.T.Urazbaev of the Academy of Sciences of the Republic of Uzbekistan, Tashkent

Abdumalik Abdixamit ugli Qayumov bachelor, Tashkent Chemical Technological Institute

\title{
PROCESSES, OCCURRED DURING DEWATERING OF MOISTURE SATURATED MATERIAL
}

\footnotetext{
Abstract: In the article reviewed modern state of research and existing models of moisture-rich material, pressed between rotating rollers.

Key words: moisture removal, hydro-dynamics, deformation, moisture-rich material, roll mechanisms.

Language: Russian

Citation: Bahadirov GA, Barakaev NR, Abdukarimov A, Umarov AA, Qayumov AA (2017) PROCESSES, OCCURRED DURING DEWATERING OF MOISTURE SATURATED MATERIAL. ISJ Theoretical \& Applied Science, 06 (50): 53-58.

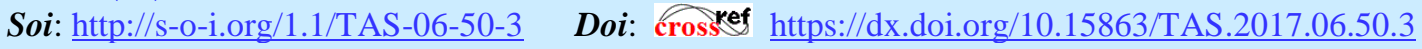

\section{ПРОЦЕССЫ, ПРОИСХОДЯЩИЕ ПРИ ОБЕЗВОЖИВАНИИ ВЛАГОНАСЫШЕННОГО МАТЕРИАЛА}

Аннотация: В статье рассматривается современное состояние исследований и существующие модели движения влагонасыщенного материала, зажатого между вращающимися валками.

Ключевые слова: удаление влаги, гидродинамика, деформацчия, влагонасыщенный материал, валковые механизмы.
}

\section{Introduction}

Механическая технология обработки различных материалов с применением валковых механизмов широко применяются во многих отраслях промышленности. В легкой, текстильной, хлопкоочистительной, пищевой, металлургической, резиновой, химической, целлюлозно-бумажной и других отраслях промышленности, валковые механизмы являются основными рабочими органами ряда технологических оборудований $[1$, с. $8-9 ; 2$, с. 3 ; 3 , c. 47].

\section{Materials and Methods}

Впервые контактная задача взаимодействия катков или цилиндров и цилиндра с плоскостью решена Г.Герцем в статике. При решении принимались условия, что, материалы контактирующихся тел однородны и изотропны, деформации тел абсолютно упруги и подчиняются закону Гука, величина площадки контакта мала по сравнению с размерами валков. При этих условиях нормальные напряжения распределяются по поверхности контакта по эллиптическому закону. Однако, в большинстве 
практических задач, связанных с обработкой валковыми парами различных материалов, принятые предпосылки при решении контактной задачи не выполняются. Материал получает не только упругую, но и пластическую деформацию, зависимость которой от напряжения имеет не линейный характер и не подчиняется закону Гука. В связи с этим, действительные закономерности распределения контактных напряжений в валковых парах с эластичными покрытиями отличаются от эллиптического закона [4, с. 241].

Основным рабочим органом валковых машин является валковая пара, контактирующаяся с обрабатываемым материалом. Технологические процессы в валковых машинах осуществляются в результате контактного взаимодействия валков с обрабатываемым материалом. Возможность интенсивного воздействия валков на материал в зоне контакта способствует повышению эффективности технологических процессов.

Научно-исследовательские работы в области валкового оборудования в основном проводились в направлении разработки методик его проектирования или совершенствования с целью создания высокопроизводительных, эффективных и экономичных машин. Они исследовались с учетом особенностей различных листовых материалов, а также процессов обработки листовых материалов с валковыми устройствами применительно к различным видам оборудования [5, с. $48 ; 6$, с. $3 ; 7$, с. 6$]$.

Для выбора оптимальных параметров проектируемой машины необходимы расчетные формулы или готовая программа, описывающая поведение исследуемого объекта и позволяющая для любого заданного набора параметров рассчитывать проектируемую систему и вычислять все критерии качества.

При исследовании валковой пары необходимо исходить из сил, действующих на валки в процессе работы. Величина и направление действующих сил, при захвате обрабатываемого материала и в установившимся режиме различны, и они зависят от многих параметров и факторов. К этим факторам относятся: диаметры валков, которые могут быть равными или различными; кинематическая связь между валками, может быть жесткой или же один из валков свободный, который будет вращаться за счет фрикции; установка валков, которые могут быть установлены горизонтально или наклонно, один над другим с расположением их осей вращения на вертикальной или на наклонной плоскости, а также вертикально; при этом для создания прижима между валками, они могут быть подвижным верхний, нижний или оба валка; валки могут быть твердыми или упругими покрытиями, которые могут быть влагопроницаемыми или не влагопроницаемыми, в зависимости от технологии могут выбираться их комбинации. Кроме того, один из валков или оба валка могут быть составными. Валки могут быть обогреваемыми или не обогреваемыми, могут быть их комбинации. Помимо того, обрабатываемый материал на выходе из валков может подвергаться растяжению с некоторой силой или заталкиванию при захвате в валки, это имеет место для твердых материалов [2, с. 84].

Проблемы

совершенствования существующих и разработки новых технологических процессов обработки материала и создания оптимальных конструкций валковых машин тесно связаны с геометрическими, кинематическими и динамическими закономерностями процесса. Эти закономерности легли в основу исследований А.П. Грудева, В.М. Клименко, А.М. Онищенко, И.Д. Кугушева, Г.А. Мюллера, Н.Е. Новикова, А. Ниссэна, Д. Свита и др. [2, с. 6].

Кинематические и динамические особенности процесса прокатки металла в различных валках достаточно хорошо изучены в предположении, что справедлив закон сухого трения Амонтона, согласно которому коэффициент трения является константой.

При исследовании движения материала, зажатого между вращающимися валками и содержащего жидкостные компоненты, существуют различные подходы. В.И. Смирновым и А.Ф.Новиковым исследовано движение продукта, зажатого между вращающимися валками, при постоянном коэффициенте трения в очаге деформации и без изменения массы рассматриваемого материалы [8, c. 15].

Г.К.Кузнецовым

рассмотрена гидродинамика процесса отжима текстильных материалов с использованием изотропной и слоистой моделей, причем принято, что движение жидкости в них подчиняется закону Дарси. При пропуске между валками, под нагрузкой отжимаемый материал деформируется, отдельные его элементы сближаются, возникает гидравлическое давление, и движение жидкости. Утверждается, что движение жидкости при отжиме осуществляется за счет гидродинамического давления, возникающего в слое обрабатываемого материала, вследствие деформации его валками. Закон распределения гидродинамического давления по длине зоны деформации практически не зависит от вида и свойств отжимаемого материала $[9$, с. 4;10, 19].

Непосредственное использование этих теорий при изучении процесса обработки различных плоских влагосодержащих 
материалов, между двумя валками дают значительную погрешность.

Значительные теоретические и прикладные разработки известны по вопросам отделки бумаги в каландрах. По теории Ниссэна в зоне контакта рабочих валков, которую он делит на две части: входную и выходную, влага из материала удаляется в выходную часть. Это объясняется тем, что удаление влаги из материала происходит под действием капиллярных сил в выходной части зоны контакта, где упругие покрытия валков при расширении впитывают влагу $[11$, с. 605].

Результаты других исследователей [12, с. 367], показывают обратную картину: влага удаляется из входной части зоны контакта и в выходной части, при этом имеет место впитывание влаги материалом из покрытий валков. Следует иметь в виду, что характер удаления влаги из материалов и впитывание влаги материалом покрытий валков зависит от свойств материалов и механических параметров валковой пары.

На основе уравнений фильтрации КозениКармана и гидростатического давления Гаскелла, разработана физическая модель процесса отжима на валковых устройствах, в основу которой положена гипотеза о том, что данный технологический процесс рассматривается как сочетание двух одновременно протекающих и взаимосвязанных механизмов, фильтрации жидкости и движения жидкой пленки по материалу. На основе анализа полученного уравнения сделан практический вывод о том, что глубина фильтрации жидкости в материале увеличивается пропорционально $\sqrt{R}(R-$ радиус валков), т.е. увеличение диаметра валковой пары способствует более полному удалению жидкости из волокнистого материала.

Для нормального протекания процессов механической обработки влагосодержащих листовых материалов, важное значение имеет содержание в нем влаги. Например, кожевенный полуфабрикат обычно после дубления и пролежки а также после красильно-жировальных процессов содержит около $70 \%$ влаги. Такое высокое содержание влаги отрицательно влияет на проведение последующих процессов, в частности строгания, двоения и сушки в наклейку. Содержание влаги в кожевенном полуфабрикате после отжима должно быть 55$60 \%$. Низкая, менее $50 \%$ влажность кожевенного полуфабриката резко снижает эффект последующих механических операций. Складки, образовавшиеся на нем после отжима, практически не разглаживаются.

Анализ полученных результатов показал, что зависимость остаточной влажности от диаметра валков при разных давлениях различна.
Установлено что при среднем удельном давлении, равном $1,5 \cdot 10^{6} \mu / \mu^{2}$ и выше, эффект обработки с увеличением диаметра повышается. Лучшие показатели были получены при отжиме влаги из ткани шкуры валками диаметров 0,30$0,35 \mathrm{M}$.

В работе утверждается, что чем меньше диаметр рабочих валков, тем больше удельное давление сжатия листового материала между ними и, следовательно, эффективнее качество обработки.

Ряд исследований зоны контакта валковых пар был направлен на создание математических моделей процессов. Анализ работы оборудования показывает, что только повышение скорости его работы как источник повышения производительности оборудования и труда в основном себя исчерпал. Акцент поиска резервов роста производительности труда, сейчас сместился в область механизации и автоматизации ручных и вспомогательных операций.

Определению влияния диаметров валков на эффективность обработки и определению его параметров посвящено много работ, так как диаметр валков определяет геометрию зоны контакта. Результаты исследований о влиянии диаметров рабочих валков на остаточную важность весьма противоречивы. От диаметра валков зависит ширина зоны контакта, время обработки, условия захвата и втягивания материала и соответственно качество его обработки, остаточная важность материала и др. [13, с. $231 ; 14$, с. 119]

На основании экспериментальных исследований В.А.Кузнецовым [15, с. 15] создана математическая регрессионная модель валкового отжима, связывающая факторы: давление, диаметры валков, скорость ткани и остаточную влажность. Предложенная модель позволяет рассчитать эффект обезвоживания и основные параметры валкового модуля. Установлено, что зависимость изменения толщины ткани от давления идентична для всего исследованного ассортимента и характеризуется наибольшим изменением толщины в диапазоне давлений до 15 МПа, относительная деформация тканей при этом составляет 56-72\%. Дальнейшее увеличение давления до 25 МПа способствует изменению относительной деформации всего на 2-4\%, также, увеличение скорости движения ткани с 2,5 до 4,2 м/с ухудшает отжимной эффект на $10 \%$. На основании исследований, автор делает вывод, что для интенсификации отжима ткани необходимо увеличить нагрузку и уменьшить диаметры валков. Для обеспечения усиленного отжима ткани оптимальным является диаметр рабочих валков 0,21-0,25 м при нагрузке 30-50 кН/м. 
Технический уровнь валковых модулей текстильного отделочного производства повышается путем создания качественно новых методик проектирования, учитывающих конструктивные особенности валков, характеристики обрабатываемого материала и параметры технологического процесса обработки ткани. На основе силового анализа валкового модуля разработан алгоритм их расчета. Установлено, что между сближением валков и интенсивностью нагрузки в зоне контакта существует нелинейная зависимость, характер которой зависит от радиусов взаимодействующих валков, толщины и модуля упругости первого рода эластичного покрытия.

Г.К.Кузнецовым и рядом автором [7, с. 6,9 , c. 13] выполнены исследования в области механики валковых механизмов. Выявлены силовые режимы работы этих механизмов для нескольких схем кинематического взаимодействия валков и обрабатываемого материала, изложены вопросы теории распределения напряжений по поверхности контактов валков при различной степени податливости покрытий. Силовой анализ валковых механизмов с учетом стационарности их работы уравновешенности звеньев проводился статистическими методами и заключался в определении главного вектора и главного момента нормальных и касательных сил. Нормальные и касательные напряжения распределяются вдоль дуги контакта в соответствии с закономерностями, которые определяются схемой действия сил, упругими и фрикционными свойствами обрабатываемого материала и покрытия валков. Рассмотрены варианты возможных законов распределения нормальных напряжений: эллиптический, параболический, гармонический и показательный. Установлено, что распределение нормальных и касательных напряжений для зоны контакта валковой пары в динамике отличается от статического и становится несимметричным.

Также Г.К.Кузнецов [9, с. 56] установил, что касательные контактные напряжения при статическом контакте и при вращении с незначительным моментом сопротивления на ведомом валке, меняет знак при переходе через осевую плоскость валков. Абсолютные величины напряжений практически симметричны относительно этой плоскости. Также рассмотрены переходные процессы, возникающие при прокатке валковой парой неравномерного слоя материала. Установлено, что нагрузки, действующие на прокатываемый материал, при больших скоростях возрастают. В результате большого объема исследований Г.К.Кузнецовым разработана методика проектирования валковых устройств текстильных машин и предложена их классификация, позволяющая систематизировать исследования в области механики валковых машин и методов расчета их рабочих органов.

Экспериментально исследовано влияние покрытий рабочих валков на эффективность удаления влаги и установлено, что при прочих равных условиях применение нежестких покрытий снижает эффект обработки, но сохраняет физико-механические свойства волокон материала. Это объясняется увеличением размера площадки контактов валков и снижением удельного давления на материал.

В настоящее время величина нагрузки на валки устанавливается на основании экспериментальных данных из-за отсутствия современных методов их расчета. Совершенствуя методы расчета вытяжных механизмов для мокрого прядения льна В.В.Фарукшин [16, с. 16] рекомендует определение нагрузки на нажимной валик с использованием дискретной модели силового взаимодействия элементов выпускной пары. При условии, что нагрузка вдоль образующей валка распределяется равномерно, задаваясь законом распределения интенсивности нагрузки вдоль оси нажимного валка и проверяя выполнение условий силового взаимодействия элементов выпускной пары, полученных автором, можно найти необходимую нагрузку на валок.

На основе гипотезы о нерастяжимости контактирующей поверхности, без учета влияния обрабатываемого материала К.Д.Буданов [17, с. 154], проводил силовой анализ эластичных покрытий валковой пары. Им получены упругие характеристики грузовиков тканепечатных машин в виде степенной функции, основные показатели процесса печати; максимальное и среднее давление в контактной полосе, исследован процесс скольжения печатных валков по грузовику.

Лодойн Удвал [18, с. 162] исследуя процесс транспортировки тканей валковыми модулями, установил, что относительная деформация покрытия валков модуля по длине дуги их контакта изменяется по параболическому закону. Динамическая нагрузка от шва на валок пропорциональна его массе, квадрату скорости движения ткани и зависит от параметров швов. Для снижения динамических нагрузок при прохождении швов рекомендуется снижение массы валков и в трех валковых модулях рекомендуется применение систем валковых модулей с двумя степенями свободы перемещения.

В технологии механической обработки различных материалов отжим влаги из влагосодержащего материала играет важную роль. Которая выполняется преимущественно на валичных машинах. Особенности процесса 
валичного отжима изучены недостаточно [19, с. 15].

Непосредственное использование вышеперечисленных теорий при изучении процесса отжима влагосодержащего материала между двумя валками дают значительную погрешность. Она возникает, в частности, из-за того, что закон сухого трения Амонтона здесь не применим, т.к. при отжиме влагосодержащего материала приходится учитывать его способность прилипания к поверхностям валков в зависимости от содержания в ней влаги. В результате при наличии двух валков коэффициенты трения будут зависеть также от продольной координаты в очаге деформации.

Механическое обезвоживание материала в промышленности экономически целесообразно, так как гораздо дешевле сушки. Но механическое обезвоживание не беспредельно. Какие бы совершенные конструкции не создавались, снизить влагосодержание материала механическим путём ниже определенного предела, зависящего от ряда факторов, невозможно.

Таким образом, научные основы движения влагонасыщенного упругопластического тела переменной массы между вращающимися цилиндрами имеющими упругие оболочки полностью не решены.

Чтобы полнее определить возможность отжимных машин, вкратце рассмотрим явления влагопоглощения и соответственно влагоотдачи материала.

Влагосодержащие материалы, используемых промышленностью, достаточно велико. Они отличаются друг от друга не только по своей структуре и химическому составу, но способности поглощать воду.

При погружении пористого материала в жидкость она в большой или меньшей степени увеличивается в своем объеме, что и является набуханием, степень которого изменяется количеством жидкости, поглощенный единичном объеме материала. Этот процесс идет до некоторого предела и зависит от температуры, с повышением которой скорость набухания растет, но абсолютная величина его понижается. Через определенное время замачивание достигается предельный объем набухания. Количество поглощенной жидкости зависит от вида и свойств материала. Некоторые естественные материалы являются гидрофильными, т.е. молекулы воды испытывают притяжение к ним и обладают способностью смачивать его.

Таким образом, чем выше способность материала к набуханию, тем больше она поглощает влаги.

Неодинаковая способность материала к адсорбции предопределяет и различную их влагоотдачу при отжиме. В материалах после их смачивания содержится влага трех видов: свободная (поверхностно - обволакивающая ), капиллярная и адсорбционная (влага набухания).

При обезвоживании влагосодержащего материала происходят следующие физические процессы. В первый период отжима удаляется поверхностно - обволакивающая влага, удерживаемая на поверхности материала или в ее порах. Затем удаляется капиллярная влага сначала из грубых капилляров, потом из мелких ультрапор. При дальнейшем обезвоживании остается лишь адсорбированная вода.

При исследовании влагоотдачи влагосодержащего материала было замечено, что сначала отдача воды идет довольно быстро, а затем, когда остается небольшое количество влаги, обезвоживание сильно замедляется.

Представим общий объем влагосодержащего материала $V$ равным сумме:

где $V_{n}$ - объем пор;

$$
V=V_{n}+V_{k},
$$

$V_{k}$ - объем занимаемый частицами материала.

Введем также обозначения:

$Q_{k}$ - масса материала;

$Q_{n}$ - масса воды в порах.

Абсолютная влажность материала материала $W=\frac{Q_{n}}{Q_{k}} 100 \%$.

Обменная масса материала

$$
\gamma_{k}=\frac{Q_{k}}{V_{k}}\left[\kappa 2 /{ }_{M^{3}}\right]
$$

Пористость $n=\frac{V_{n}}{V_{k}}$.

Влагопроводность в процесс отжима не остается величиной неизменной, так как при сжатии прессуемой массы материала объем пор сокращается и резко меняется сопротивление движению жидкости. Величина $n$, характеризующая пористость материала, представляет собой отношение объема полостей, приходящихся на пустоты, занятие жидкостью, к объему сухой массы материала. Очевидно, что

$$
n=f\left(P_{T}\right) \text {, }
$$

где $P_{T}-$ часть внешнего давления, воспринимаемая влагосодержащим материалом.

При повышении давления масса влагосодержащего материала сжимается и пористость его снижается. Мерой способности деформироваться является отношение

$$
a=-\frac{d n}{d p},
$$

причем для различных влагосодержащего материала величина «a» будет различна. 


\begin{tabular}{|c|c|c|c|c|c|c|}
\hline Impact Factor: & $\begin{array}{l}\text { ISRA (India) } \\
\text { ISI (Dubai, UAE } \\
\text { GIF (Australia) } \\
\text { JIF }\end{array}$ & $\begin{array}{l}=1.344 \\
=0.829 \\
=0.564 \\
=1.500\end{array}$ & $\begin{array}{l}\text { SIS (USA) } \\
\text { PИНЦ (Russia } \\
\text { ESJI (KZ) } \\
\text { SJIF (Morocco }\end{array}$ & $\begin{array}{l}=0.912 \\
=\mathbf{0 . 2 3 4} \\
=\mathbf{3 . 8 6 0} \\
=\mathbf{2 . 0 3 1}\end{array}$ & $\begin{array}{l}\text { ICV (Poland) } \\
\text { PIF (India) } \\
\text { IBI (India) }\end{array}$ & $\begin{array}{l}=6.630 \\
=1.940 \\
=4.260\end{array}$ \\
\hline
\end{tabular}

\section{Conclusion}

Итак, влагопроводность и пористость зависят от давления. И та и другая величины с повышением давления снижаются. Но этот процесс не бесконечен. Существует такое предельное значение давления, при котором пористость уже не изменяется, а материал становится практически водонепроницаемым.

\section{References:}

1. Klinkov AS, Kochetov VI, Sokolov MV, Beljaev PS, Odnol'ko VG (2005) Proektirovanie i raschet valkovyh mashin dlja polimernyh materialov. Tambov, Izd. TGTU, 2005. - $128 \mathrm{p}$.

2. Bahadirov GA (2010) Mehanika otzhimnoj valkovoj pary. Tashkent, Fan, 2010. - 156 p.

3. Ivanov VA, Rashkin VV (2012) Tendencii i perspektivy razvitija oborudovanija kozhevennyh proizvodstv. Jelektrotehnicheskie i informacionnye kompleksy i sistemy № 1, t. 8, 2012. 47-52 p.

4. Jeyapalina S, Attenburrow GE, Covington AD (2007) Dynamic Mechanical Thermal Analysis (DMTA) of leather - Part 1: Effect of tanning agent on the glass transition temperature of collagen, Journal- Society of Leather Technologists and Chemists. 08/2007; 91(6):236-242.

5. Abdukarimov A (2012) Klassifikacija valkovyh modulej. // Problemy mehaniki. Tashkent, 2012. -№4, -p.48-52.

6. Pusheng C, Thomas A, Louella AP, Ashford AG, Paul SS (2014) Method of processing leather material. United States Patent. US 8,776,554 B2. Jul. 15, 2014.

7. Fomin JuG, Kuznecov GK, Kiselev IA (1994) Konstrukcija i raschet mehanizmov valkovyh mashin dlja obrabotki tkanej. Ivanovo.: Iv. Gos. tekstil'naja akademija, 1994. - 196 p.

8. Smirnov VI, Novikov AF (1975) Issledovanie dvizhenija produkta, zazhatogo mezhdu vrashhajushhimisja valkami. Tehnologija tekstil'noj promyshlennosti 1975. №1.

9. Kuznecov GK (2000) Mehanika tekstil'nyh mashin $\mathrm{v}$ primerah $\mathrm{i}$ zadachah: Uchebn. posobie. - Kostroma: KGTU, 2000. - 68 p.

10. Darda IV (2004) Razrabotka teoreticheskih osnov sovershenstvovanija tehnologicheskogo oborudovanija kozhevenno-mehovogo proizvodstv. Diss. ... dokt. tehn. nauk. Moscow. 2004. 325 p.
11. Nissan A (1954) Functions of the felts in water removal on the papermaking machine - Tappi, 1954, 37 n12, p. 597-606.

12. Sweet JA (1961) Basic study of water removal at the press - Pulp and paper magazine of Canada, 1961, 62 n 7, p. 367.

13. Polomoshnyh SP, Radnaeva VD, Titov OP, Kalashnikova JM (2013) Osnovy mehanicheskoj tehnologii obrabotki kozhevenno-mehovogo polufabrikata na zhidkostnyh operacijah. Visnik Hmel'nic'kogo nacional'nogo universitetu №3, 2013. p. 226232.

14. Bahadirov GA (2016) Sovershenstvovanie tehniki i tehnologii mehanicheskoj obrabotki kozhevennogo polufabrikata. Izvestija Kyrgyzskogo gosudarstvennogo tehnicheskogo universiteta im. I.Razzakova. Tom: 37. 1, 2016. p. 118-131.

15. Kuznecov VA (1984) Obosnovanie konstruktivnyh parametrov vysokoproizvoditel'nyh valkovyh mashin intensivnogo otzhima: Avtoref. diss ... k.t.n. Kostroma, 1984. $-18 \mathrm{p}$.

16. Farukshin VV (1999) Sovershenstvovanie metodov rascheta vytjazhnyh mehanizmov dlja mokrogo prjadenija l'na: Avtoref. diss. ... k.t.n. - Kostroma, KGTU, 1999. - 19 p.

17. Budanov KD (1961) Nekotorye voprosy mehaniki tkanepechatnyh mashin: Avtoref. diss. ... k.t.n. - M., 1961. - 192 p.

18. Lodojn Udval (1998) Optimizacija processa transportirovki tkanej valkovymi moduljami i razrabotka uslovij snizhenija jenergozatrat na ih privod: Diss. ... kand. tehn. nauk. - Ivanovo: Iv. Gos. Tekstil'naja akademija, 1998. - 194 p.

19. Bahadirov GA (2011) Investigation of motion of water-containing sheet material in-between rotating pair of rollers. International Journal of Modern Manufacturing Technologies, Vol. III, No. 1/2011. p. 15-20. 